\title{
Síndrome de Burnout em Professores de Educação Pré-Escolar
}

\author{
Cristina Delgado Rodrigues \\ Laura Brandão Chaves \\ Mary Sandra Carlotto \\ Universidade Luterana do Brasil
}

\begin{abstract}
RESUMO
Professores, atualmente, estão mais expostos a diversos estressores ocupacionais que, se persistentes, podem levar à síndrome de burnout, fenômeno psicossocial constituído por três dimensões: Exaustão Emocional, Despersonalização e Baixa Realização Profissional. O objetivo deste estudo foi verificar a existência de associação entre as dimensões da síndrome de burnout e variáveis demográficas, laborais e psicossociais em uma amostra de 34 professores de educação pré-escolar da cidade de Porto Alegre. Foram utilizados como instrumentos de pesquisa o Maslach Burnout Inventory e um questionário de dados sociodemográficos e laborais para as demais variáveis. Resultados revelam baixos índices nas dimensões de exaustão emocional e despersonalização e alto índice na dimensão de realização profissional. Com relação às variáveis demográficas somente a variável ter companheiro associou-se à dimensão de exaustão emocional, sendo que as professoras que possuem companheiro apresentaram média mais elevada. Nas variáveis laborais e psicossociais, resultados obtidos através da prova de correlação de Pearson e $t$ de Student evidenciam que quanto maior a carga horária, o número de alunos atendidos diariamente, o tempo de docência e o pensamento de mudar de profissão, maior é a exaustão emocional. Já a realização profissional diminui à medida que aumenta a frequência do pensar em mudar de profissão.
\end{abstract}

Palavras-chave: síndrome de burnout; professores; pré-escola.

\section{ABSTRACT \\ Burnout Syndrome in preschool teachers}

Nowadays, teachers are more exposed to several occupational stress factors, which if persistent may lead to Burnout Syndrome, a psychosocial phenomenon constituted by three dimensions: emotional exhaustion, depersonalization and reduced professional achievement. The aim of this study was to verify the existence of association among the Burnout Syndrome dimensions and demographic, labor and psychosocial variables in a sample of 34 preschool teachers in the city of Porto Alegre, Brazil. The MBI - Maslach Burnout Inventory - was used along with a questionnaire to assess other socio-demographic and labor variables. The results revealed low rates of the emotional exhaustion and depersonalization dimensions, and a high rate of Professional Achievement. As for demographic variables, only "having a partner" associated to the emotional exhaustion dimension, in which the teachers who have partners presented a higher mean. Regarding labor and psychosocial variables, results obtained using Pearson's correlation and the Student's $t$-test showed that as working hours, number of students who are taken care of daily, time of teaching practice and thoughts of changing career increase, the emotional exhaustion increases as well. Professional achievement decreases as the frequency of thinking of changing career increases.

Keywords: burnout syndrome; teachers; preschool.

O Ministério da Saúde do Brasil (2001) reconhece a Síndrome de Burnout (SB) ou Síndrome do Esgotamento Profissional como um tipo de resposta prolongada a estressores emocionais e interpessoais crônicos no trabalho. Burnout é considerado um dos agravos ocupacionais de caráter psicossocial mais importante na sociedade atual (Salanova \& Llorens, 2008; Schaufeli, Leiter \& Maslach, 2009). Acomete, principalmente, profissionais da área de serviços ou cuidadores, quando em contato direto com os usuários, como os traba- 
lhadores da educação, da saúde, policiais, assistentes sociais, agentes penitenciários, entre outros.

Seligmann-Silva (1994) refere-se ao burnout como uma síndrome especial de esgotamento profissional, sendo esta um conjunto de respostas às situações estressantes, próprias da atuação no trabalho, cuja especificidade está na necessidade de interagir e do cuidado constante com outras pessoas. Logo, a natureza do trabalho se constitui na fonte de tensões que proporcionará aos sujeitos experiências estressoras, exigindo reações específicas e estereotipadas, manifestando-se com atitudes desfavoráveis em relação ao trabalho, perda de interesse em relação aos clientes e desenvolvimento de imagens negativas sobre si mesmo.

Segundo Carlotto (2002), atualmente, a definição mais aceita de burnout é a fundamentada na perspectiva social-psicológica, sendo esta constituída de três dimensões: exaustão emocional, despersonalização e baixa realização no trabalho. Maslach, Schaufeli e Leiter (2001) definem as três dimensões da síndrome: exaustão emocional, caracterizada por uma falta ou carência de energia, entusiasmo e um sentimento de esgotamento de recursos; despersonalização, que se caracteriza por tratar os clientes, colegas e a organização como um objeto; e baixa realização no trabalho, que é a tendência do trabalhador em se autoavaliar de forma negativa. Os trabalhadores sentem-se infelizes e insatisfeitos com seu desenvolvimento profissional.

O processo de desenvolvimento do burnout é individual e sua evolução pode levar anos e até mesmo décadas (Rudow, 1999). Seu surgimento é paulatino, cumulativo, com incremento progressivo em severidade (França, 1987), não sendo percebido pelo indivíduo em sua fase inicial, que, geralmente, recusa-se a acreditar estar acontecendo algo errado com ele (França, 1987; Rudow, 1999). No que diz respeito às suas repercussões, Codo (1999) destaca as doenças cardiovasculares, distúrbios advindos do estresse, labirintite, faringite, neuroses, fadiga, insônia e tensão nervosa. Conforme o autor, o estresse ocupacional pode ser constatado, entre os docentes, pelos seus problemas de saúde e pela redução na frequência ao trabalho. Além das consequências individuais, Abel e Sewell (1999) referem que o burnout em professores afeta diretamente o trabalho com alunos e a qualidade de ensino.

Segundo Rosales-Álamo, Jiménez-Betancort e Serio-Hernández (2007), diferentes estudos têm demonstrado que a crescente pressão e demanda social, associadas com o aumento da dificuldade no processo educativo, repercutem no trabalho do professor, afetando de forma crítica seus sentimentos, percepções, estado psicológico, imagem social e satisfação com seu trabalho. Na visão de Farber (1991), do ponto de vista público, a categoria de professores sofre muitas críticas, é extremamente cobrada em seus fracassos e raramente é reconhecida por seu sucesso. $\mathrm{O}$ autor destaca que, mesmo que esta seja uma tendência de todas as profissões, nenhuma categoria tem sido tão severamente avaliada e cobrada pela população em geral nas últimas duas décadas como a de professores.

No Brasil, de acordo com Reis, Araújo, Carvalho, Barbalho e Silva (2006), há relativa escassez de estudos sobre a saúde do professor em comparação com trabalhadores de outras profissões. Com relação ao burnout, o mesmo ocorre, no entanto, já se identificam alguns estudos sobre essa temática em professores (Batista, 2010; Carvalho, 1995; Carlotto, 2002; Carlotto \& Câmara, 2007; Codo, 1999; Mallar \& Capitão, 2004; Moura, 1997; Silva \& Carlotto, 2003). Ao verificar o estado da arte da síndrome de burnout no Brasil, Carlotto e Câmara (2008) identificaram 28 artigos nacionais sobre a síndrome sendo que, destes, somente 9 artigos $(32,2 \%)$ foram realizados com professores. Não foram identificados artigos lidando com o nível de ensino infantil.

De acordo com Araújo, Carvalho, Porto, Reis e Silvany Neto (2005), a categoria docente é uma das mais expostas a ambientes conflituosos e de alta exigência de trabalho. Assim, estressores psicossociais estão constantemente presentes e atuando sobre a saúde do professor. Segundo Farber (1991), as manifestações de burnout em professores podem ser divididas em sintomas individuais e profissionais, destacando, entretanto, que estas questões são de difícil generalização e descrição universal. Em geral, segundo o autor, os professores sentem-se emocional e fisicamente exaustos, estão frequentemente irritados, ansiosos, com raiva ou tristes. As frustrações emocionais peculiares a este fenômeno podem levar a sintomas psicossomáticos como insônia, úlcera, dores de cabeça e hipertensão, além do uso abusivo de álcool e medicamentos, incrementando problemas familiares e conflitos sociais. Nos aspectos profissionais, o professor pode apresentar prejuízo no seu planejamento de aula, tornando-se menos frequente e cuidadoso; apresentar perda de criatividade e entusiasmo, sentir menos simpatia pelos alunos e menos otimismo quanto à avaliação do seu futuro; pode sentir-se facilmente frustrado pelos problemas ocorridos em sala de aula.

O cenário educativo brasileiro apresenta um quadro bastante problemático no que se refere às questões relacionadas à saúde dos professores e às condições de tra- 
balho (Mariano \& Muniz, 2006). Ensinar é uma atividade, em geral, altamente estressante, com repercussões evidentes na saúde física, mental e no desempenho profissional dos professores (Reis \& cols., 2006). Assiste-se, hoje, a um processo de desvalorização dessa profissão acompanhado da expansão das funções docentes (Noronha, Assunção \& Oliveira, 2008).

No trabalho docente, conforme Carlotto (2002), alguns estressores são típicos da natureza da função e outros são ocasionados pelo contexto onde essa se realiza. Segundo Leite e Souza (2007), no que se refere aos problemas relacionados às condições de trabalho, há consenso, na bibliografia, quanto aos diversos problemas enfrentados pelos professores. Os autores referem estudos nos quais são destacados aspectos como: precárias condições de trabalho (temperatura, ruído, iluminação, espaço físico, material didático); organização do trabalho (multiplicidade de tarefas diferenciadas e simultâneas, ritmos de trabalho, pouca frequência de pausas, os movimentos repetitivos, o aumento das exigências cognitivas, mobilização da subjetividade, capacidade de diagnosticar e de decidir, perda de autonomia, burocratização e rotinização das atividades educativas, a prescrição do trabalho, a padronização do currículo e dos métodos de ensino, a falta de acompanhamento técnico, novas exigências de qualificação, como polivalência, qualificação técnica, realização de horas extras); físicos (posturas desconfortáveis, uso elevado e frequente da voz, peso de grandes quantidades de materiais).

Sparks (1983) entende que o estresse neste tipo de profissão ocorre porque os professores ficam expressiva parte de seu horário de trabalho fisicamente isolados de seus colegas de trabalho, não podendo expressar e dividir suas ideias com eles. Muitos professores não visualizam perspectivas em seu trabalho, não examinam seu sucesso profissional, sua competência e nem a satisfação que obtém com o serviço. Trabalham estabelecendo uma rotina, esquecendo-se de atividades extraprofissionais e de lazer, não criando um estilo de vida saudável. Harden (1999) acrescenta a esses estressores a preocupação em como fazer seu trabalho de forma a lidar com a pressão para a conformidade e a necessidade de adotar algumas práticas com as quais o professor não se identifica.

Marques (1979) aponta que o estresse dos professores de educação infantil é decorrente de situações externas como: os baixos salários levando a necessidade de trabalhar em mais de uma escola ou mesmo ter outro tipo de atividade não docente; falta de preparo ou treinamento adequado ao nível e ao tipo de criança que ensinam; ausência de supervisão pedagógica e controle administrativo por parte da escola; deficiência de condições materiais, como espaço físico e recursos de ensino-aprendizagem; falta de status social para o professor, por desvalorização de sua atividade pelos pais e outros membros representativos da sociedade. Esse autor também menciona que outros educadores e estudiosos do assunto vêem, contudo, como mais sérios os aspectos intrínsecos da profissão docente como causas mais plausíveis do estresse. Entre eles poderiam ser citados como mais frequentes: a insatisfação pessoal quanto ao seu modo de vida; falta de motivação para a busca de uma atividade mais dinâmica e envolvente; carência afetiva; componentes de agressividade não suficientemente elaborados e canalizados para uma ação construtiva; tristeza e solidão.

Segundo Mizukami e Reali (2002), há muitos problemas enfrentados na área da educação infantil, que compreendem desde a estrutura física das creches até a formação de seus profissionais. Diante deste quadro, se o professor de educação infantil não tiver clareza do sentido das atividades por ele desenvolvidas com as crianças e, ainda, se ele não identificar, nas suas ações, qual a intencionalidade que está imprimindo a essa atividade, ele pode adotar como concepção de trabalho a mais cômoda em relação ao contexto em que exerce a sua função.

O trabalho de cuidar, realizado pelo professor de creche, é complexo e requer habilidades de ordem psicológica, organizativa e estratégica. Requer rapidez de decisão, capacidade de expressão, de combinação de recursos pedagógicos, da disponibilidade representada pelo sorriso acolhedor e, ao mesmo tempo, agilidade para deslocar-se de um lugar a outro e uma capacidade singular para escutar, sentir e compartilhar com o outro (Marques, 1979).

Assim, considerando que burnout é um fenômeno psicossocial decorrente de estressores laborais, este estudo observacional analítico transversal (Grimes \& Shulz, 2002) procurou identificar a existência de associação entre as dimensões da SB e variáveis demográficas, laborais e psicossociais em professores de educação pré-escolar.

\section{MÉTODO}

\section{Participantes}

Participaram do estudo 34 professores de ensino pré-escolar pertencentes a seis escolas privadas de um bairro de classe média da cidade de Porto Alegre e que atuam na educação pré-escolar há, no mínimo, um ano. 
Os professores, em sua totalidade, são do sexo feminino. Em sua maioria, são casados $(52,9 \%)$, possuem filhos $(52,9 \%)$ e a média de idade é de 30,65 anos $(\mathrm{DP}=8,52)$. Quanto à formação profissional, a maioria dos professores possui magistério $(55,9 \%)$, superior completo $20,6 \%$ e pós-graduação $23,5 \%$. Grande parcela dos professores trabalha nos turnos manhã/tarde $(82,4 \%)$ e $11,8 \%$ trabalham no turno da tarde e $5,9 \%$ no turno da manhã. No que diz respeito ao vínculo institucional, a maioria dos docentes $(87,9 \%)$ trabalha somente em uma escola. Desenvolvem suas atividades em uma carga horária média de 40,65 horas $(\mathrm{DP}=7,41)$, atendendo diariamente uma média de 21,36 alunos $(\mathrm{DP}=15,01)$. Com relação ao tempo de docência, apresentam uma média de 8,15 anos $(\mathrm{DP}=7,82)$, na escola atual 4,75 anos $(\mathrm{DP}=3,96)$ e no nível de ensino 6 anos ( $\mathrm{DP}=4,21)$.

\section{Instrumentos}

Para o levantamento das variáveis sociodemográficas e laborais, foi elaborado um instrumento com base na revisão bibliográfica sobre a SB em professores. Para avaliar a SB foi utilizado o MBI - Maslach Burnout Inventory - elaborado por Maslach e Jackson (1986), versão específica para professores, tradução e adaptação para o uso no Brasil realizada por Carlotto e Câmara (2004). As autoras apontam que a versão brasileira do MBI demonstrou os requisitos necessários em termos de consistência interna e validade fatorial para ser utilizada na avaliação da SB em professores em nossa realidade. A frequência das respostas é avaliada através de uma escala de pontuação que varia de 0 a 6 . Utilizamos 0 para "nunca", 1 para "quase nunca", 2 para " algumas vezes", 3 para "regularmente", 4 para "bastante vezes", 5 para " quase sempre" e 6 para "sempre".

TABELA 1

Médias e Desvio-Padrão das Dimensões de Burnout

\begin{tabular}{lcc}
\hline Dimensões & M & DP \\
\hline Exaustão emocional & 1,50 & 0,92 \\
Despersonalização & 0,63 & 0,65 \\
Realização Profissional & 4,42 & 0,90 \\
\hline
\end{tabular}

Os resultados obtidos através da correlação de Pearson evidenciam que quanto maior a carga horária, o número de alunos atendidos diariamente, o tempo de docência e o pensamento de mudar de profissão, maior

\section{Procedimentos}

Primeiramente, foi realizado contato com a diretora de cada escola e apresentado o objetivo do estudo a fim de obter a autorização e o apoio para a aplicação dos instrumentos. Após autorização, foi feito contato direto com os professores. Em cada escola, os questionários foram aplicados em grupo e recolhidos ao final do preenchimento. A aplicação foi realizada no mês de setembro de 2007. Foram realizados os procedimentos éticos conforme legislação do Ministério da Saúde (1997), resolução 196 do Conselho Nacional de Saúde (CNS). Foi esclarecido aos professores e aos diretores das instituições de ensino tratar-se de uma pesquisa sem quaisquer efeitos avaliativos individuais e/ou institucionais e que as respostas e os dados referentes aos resultados das escolas seriam anônimos e confidenciais.

O Banco de Dados foi digitado e posteriormente analisado em pacote estatístico. Primeiramente, foram realizadas análises descritivas de caráter exploratório a fim de avaliar casos omissos, identificação de extremos e possíveis erros de digitação. A análise dos dados foi realizada através da prova de correlação de Pearson para analisar a magnitude e a direção do relacionamento entre as dimensões da SB e variáveis quantitativas e a prova $t$ de Student para variáveis qualitativas.

\section{RESULTADOS}

Os resultados obtidos indicam elevado índice de Realização Profissional $(4,42)$, índices baixos de Exaustão Emocional $(1,50)$ e de Despersonalização $(0,63)$. Considerando uma escala de pontuação de 0 a 6 , conforme Tabela 1 . é a exaustão emocional. Já a realização profissional diminui à medida que aumenta a frequência de pensar em mudar de profissão, de acordo com a Tabela 2. 
TABELA 2

Matriz de Correlação entre Dimensões de Burnout e Estratégias de Enfrentamento

\begin{tabular}{lccc}
\hline Variáveis & EE & DE & RP \\
\hline Idade & 0,163 & $-0,263$ & 0,032 \\
Carga horária & $0,390^{*}$ & $-0,148$ & $-0,013$ \\
Alunos que contata diariamente & $0,476^{* *}$ & 0,069 & $-0,143$ \\
Horas de trabalho em casa & $-0,267$ & $-0,185$ & 0,298 \\
Tempo de docência & $0,383^{*}$ & $-0,053$ & $-0,136$ \\
Tempo na escola & 0,310 & 0,006 & $-0,152$ \\
Tempo no nível & 0,151 & $-0,129$ & 0,011 \\
Estresse profissão & 0,335 & $-0,171$ & $-0,113$ \\
Mudar de profissão & $0,382^{*}$ & 0,205 & $-0,490^{* *}$ \\
\hline
\end{tabular}

Nota: ** Correlação significativa ao nível de 1\%; * Correlação significativa ao nível de 5\%;

EE: Exaustão emocional; DE: Despersonalização; RP: Realização profissional.

Com relação à análise realizada com a prova $t$ de Student, verifica-se diferença somente para a variável ter companheiro. Assim, professoras que possuem companheiro têm média de exaustão emocional mais elevada que as colegas que não possuem $(\mathrm{p}=0,024)$. Ter filhos e formação educacional não se associou a nenhuma das dimensões de burnout.

\section{DISCUSSÃO}

Os resultados obtidos indicam elevado índice de Realização Profissional e índices baixos de Exaustão Emocional e Despersonalização. Assim, pode-se afirmar que o grupo não apresenta indicadores da SB, pois, segundo Maslach e Jackson (1981), altos escores em exaustão emocional e despersonalização e baixos escores em realização profissional são indicativos de burnout.

É importante destacar o elevado índice obtido na dimensão Realização Profissional. Esse resultado pode estar relacionado à faixa etária dos alunos de pré-escola. Segundo Reis (2005), a interação professor-aluno nesse nível de ensino é um processo relacional perpassado pela afetividade, por sentimentos e emoções. Em estudo realizado pelo autor, 94\% dos 60 alunos investigados em escolas referem à experiência pré-escolar como positiva, sendo que $90 \%$ percebem o professor como uma pessoa que tem possibilidades de criar ambiente favorável a uma experiência pré-escolar bem-sucedida, não apenas no que se refere à sua interação com os alunos, carregada de afetividade, mas também ao seu papel de profissional e de educador, ao criar as condições facilitadoras do acesso a vivências como os conhecimentos socioculturais valorizados socialmente e ao criar ambiente e espaço para a concretização dessas experiências.

O profissional da educação infantil potencializa o fator emocional, já que envolve relações interpessoais com crianças bem pequenas, o que torna ainda mais paradoxal a cisão entre o cuidar e o educar (Montenegro, 2005). Nesse sentido, pode-se pensar que nessa relação diferenciada do professor de outros níveis de ensino exista uma maior recompensa afetiva. Outra questão pode estar vinculada à opção profissional, uma vez que esse é um nível de ensino, no qual o professor ingressa por gostar de trabalhar especificamente com essa faixa etária. Este resultado confirma estudos desenvolvidos por Byrne (1991), Correia, Gomes e Moreira (2010) e Russell, Altmaier e Van Velzen (1987) que encontraram menores índices de burnout em professores que atuavam com crianças quando comparados aos que lidavam com níveis de ensino com adolescentes e estudantes universitários. Segundo Woods (1999), a pressão no trabalho é menor em professores que atendem crianças de 4 a 7 anos do que em professores que atendem crianças de 8 a 12 anos.

Professores reagem de formas diferentes frente aos mais variados fatores de estresse; contudo, para Woods (1999), está na relação aluno-professor a maior fonte de oportunidade de estresse, bem como de grandes oportunidades de recompensas e gratificações. Segundo Farber (1999), os professores, como todas as pessoas, precisam sentir-se importantes, amados e de alguma forma especiais. Eles necessitam ter estas necessidades afirmadas por quem eles trabalham. 
Em relação às variáveis demográficas, verifica-se que mulheres que possuem companheiro apresentam maior Exaustão Emocional, podendo esse resultado sugerir questões relacionadas à dupla jornada de trabalho. Apple (1995) pontua que os problemas referentes ao trabalho enfrentados pelas mulheres não são os mesmos enfrentados pelo trabalhador do sexo masculino. Ao ingressar no mercado de trabalho, a mulher passou a desenvolver uma dupla jornada, a profissional e a doméstica. As mulheres têm, hoje, uma relação dupla com o trabalho assalariado. Elas são, ao mesmo tempo, trabalhadoras remuneradas e não remuneradas. Segundo Martínez, Vera, Paterna e Alcázar (2002), a carreira profissional da mulher ainda é muito marcada pelo estresse, devido aos vários papéis que ela tem que desempenhar paralelamente a sua vida profissional.

Quanto às variáveis profissionais, evidencia-se que quanto maior a carga horária e maior o número de alunos atendidos diariamente, maior é a Exaustão Emocional. Esse resultado pode estar relacionado às características do trabalho docente nesse nível de ensino. A atuação do professor de pré-escola é bastante complexa, pois lhe exige muita atenção e cuidados, ou seja, atividades que demandam esforço físico e emocional. O trabalho de cuidar e educar crianças requer habilidades de ordem psicológica, organizativa e estratégica. Requer rapidez de decisão, capacidade de expressão, de combinação de recursos pedagógicos, da disponibilidade representada pelo sorriso e pelo acolhimento e, ao mesmo tempo, agilidade para deslocar-se de um lugar a outro e uma capacidade singular para escutar, sentir e compartilhar com o outro (Marques, 1979).

Chama atenção à associação entre as variáveis pensar em mudar de profissão, exaustão emocional e realização profissional. O resultado revela que quanto mais frequente é o pensamento de mudar de profissão, maior é o sentimento de desgaste emocional e de que o trabalho não é elemento de realização profissional. Essa variável, em alguns estudos, tem sido considerada uma consequência do burnout (Schwab \& Iwanicki, 1982; Yong \& Yue, 2007). Na medida em que o professor sente-se exausto e não realizado profissionalmente, passa a considerar a possibilidade de mudar de profissão.

Os resultados indicam ações de prevenção nos professores com maior carga horária, com maior tempo de docência e que atendem maior número de alunos. Assim, pode-se hipotetizar que esse perfil de risco pode levar o professor ao pensamento de abandonar sua profissão. Não se avalia o grupo com indicativos de burnout, mas preocupa as variáveis que se associam às suas dimensões, principalmente à exaustão emocional, pois, segundo o modelo processual de Maslach (1982), esta é a primeira dimensão a surgir, geralmente relacionada às excessivas demandas provenientes do exercício do trabalho. Depois, como estratégia defensiva ocorre o afastamento psicológico do profissional de sua clientela, surgindo, então, a fase e dimensão subsequente, a despersonalização. A última fase emergiria como resultado de todo este funcionamento, sendo caracterizada pelo sentimento de incompetência e inadequação profissional, denominada de baixa realização profissional.

O estudo aponta para a necessidade de aprofundamento dos resultados obtidos, tendo em vista que os mesmos não podem ser generalizados. São importantes novos estudos com amostras probabilísticas e em contextos educacionais variados, a fim de ampliar a compreensão sobre esse fenômeno psicossocial e sua relação com outras variáveis nesse nível de ensino ainda pouco explorado. Nesse sentido, poder-se-á subsidiar ações de prevenção por parte dos profissionais da área da saúde, comunidade escolar e a sociedade, tendo em vista as graves consequências individuais, institucionais e sociais resultantes do burnout.

\section{REFERENNCIAS}

Abel, M. H., \& Sewell, J. (1999). Stress and burnout in rural and urban secondary school teachers. The Journal of Educational Research, 92(5), 287-301.

Araújo, T. M., Carvalho, F. M., Porto, L. A., Reis, E. J. F. B., \& Silvany Neto, A. M. (2005). Trabalho e distúrbios psíquicos em professores da rede municipal de Vitória da Conquista, Bahia, Brasil. Cadernos de Saúde Pública, 21(5), 1480-1490.

Apple, M. W. (1995). Trabalho docente e textos: Economia política das relações de classe e gênero em educação. Porto Alegre: Artes Médicas.

Batista, J. B. V. (2010). Síndrome de Burnout em professores do ensino fundamental: Um problema de saúde pública não percebido. Tese de doutorado não-publicada. Fundação Oswaldo Cruz, Recife.

Byrne, B. (1991). Burnout: Investigating the impact of background variables for elementary, intermediate, secondary, and university educators. Teaching \& Teacher Education, 7(2), 197-209.

Carlotto, M. S. (2002). A síndrome de burnout e o trabalho docente. Psicologia em Estudo, 1, 21-29.

Carlotto, M. S., \& Câmara, S. G. (2004). Análise fatorial do Malasch Burnout Inventory (MBI) em uma amostra de professores de instituições particulares. Psicologia em Estudo, 9, 499-505.

Carlotto, M. S., \& Câmara, S. G. (2007). Preditores da síndrome de burnout em professores. Revista de Psicologia Escolar e Educacional, 11(1), 121-132. 
Carlotto, M. S., \& Câmara, S. G. (2008). Análise da produção científica sobre a síndrome de burnout no Brasil. PsicoPUCRS, 39, 152-158.

Carvalho, M. M. B. (1995). O professor: Um profissional, sua saúde e a educação em saúde na escola. Tese de doutorado não-publicada. Faculdade de Saúde Pública, Universidade de São Paulo, São Paulo.

Codo, W. (1999). Educação: Carinho e trabalho. Rio de Janeiro: Vozes.

Correia, T., Gomes, A. R., \& Moreira, S. (2010). Stresse ocupacional em professores do ensino básico: Um estudo sobre as diferenças pessoais e profissionais. Em Associação Portuguesa de Psicologia (Org.), Actas do VII Simpósio Nacional de Investigação em Psicologia (pp. 1477-1493). Lisboa: Associação Portuguesa de Psicologia.

Farber, B. A. (1991). Crisis in education. Stress and burnout in the American teacher. São Francisco: Jossey-Bass.

Farber, B. A. (1999). Inconsequentiality - The key to understanding teacher burnout. Em R. Vanderbergue \& M. A. Huberman (Orgs.), Understanding and preventing teacher burnout: A source book of international practice and research (pp. 159165). Cambridge: Cambridge University Press.

França, H. H. (1987). A síndrome de "burnout". Revista Brasileira de Medicina, 8(1), 197-199.

Grimes, D. A. \& Shulz, K. F. (2002). An overview of clinical research: The lay of the land. The Lancet, 359, 57-61.

Harden, R. M. (1999). Stress, pressure and burnout in teachers: Is the swan exhausted? Medical Teacher, 21, 245-252.

Leite, M. P., \& Souza, A. N. (2007). Condições do trabalho e suas repercussões na saúde dos professores da educação básica no Brasil. São Paulo: Fundacentro/Unicamp.

Mallar, S. C., \& Capitão, C. G. (2004). Burnout e hardiness: um estudo de evidência de validade. Psico-USF, 9 , 19-29.

Mariano, M. S. S; Muniz, H. P. (2006). Trabalho docente e saúde: $\mathrm{O}$ caso dos professores da segunda fase do ensino fundamental. Estudos e Pesquisas em Psicologia, 6, 76-88.

Marques, J. C. (1979). Ajudando a criança a crescer. Porto Alegre: Globo.

Maslach, C., \& Jackson, S. E. (1981). The measurement of experienced burnout. Journal of Occupational Behavior, 2, 99-113.

Maslach, C. (1982). Burnout: The cost of caring. Englewood Cliffs: Prentice- Hall

Maslach, C., \& Jackson, S. E. (1986). Maslach Burnout Inventory. Palo Alto: Consulting Psychology Press.

Maslach, C., Schaufeli, W. B., \& Leiter, M. P. (2001). Job burnout. Annual Review Psychology, 52, 397-422.

Martínez, C., Vera, J. J. Paterna, C., \& Alcázar, A. R. (2002). Antecedentes del conflicto interrol y su relación con el autoesquema de género. Anales de Psicología, 18(2), 305-317.

Ministério da Saúde. (1997). Conselho Nacional de Saúde. Diretrizes e normas para pesquisa envolvendo seres humanos. Resolução CNS 196/196. Brasília: Ministério da Saúde.

Ministério da Saúde. (2001). Doenças relacionadas ao trabalho: Manual de procedimentos para os serviços de saúde. Brasília: Ministério da Saúde; OPAS/OMS.
Mizukami, M. G., \& Reali, M. M. (2002). Formação de professores, práticas pedagógicas e escola. São Carlos: EdUFSCar.

Montenegro, T. (2005). Educação infantil: A dimensão moral da função de cuidar. Psicologia da Educação, 20, 77-101.

Moura, E. P. G. (1997). Saúde mental e trabalho: Esgotamento profissional em professores da rede de ensino particular de Pelotas - RS. Dissertação de mestrado não-publicada. Pontifícia Universidade Católica do Rio Grande do Sul, Porto Alegre.

Reis, E. J. F. B., Araújo, T. M. DE, Carvalho, F. M., Barbalho, L., \& Silva, M. O. (2006). Docência e exaustão emocional. Educação e Sociedade, 27(94), 229-253.

Reis, R. M. C. (2005). A pré-escola na visão de crianças de $1^{\mathrm{a}}$ série. Psicologia da Educação, 20, 55-75.

Rosales-Álamo, M., Jiménez-Betancort, H., \& Serio-Hernández, A. (2007). Riesgo de trastorno psicopatológico en docentes y autopercepción de bajo bienestar laboral. Retirado em 14 de novembro de 2007, de http://psiquiatria.com/articulos/estres/ 29440/

Rudow, B. (1999). Stress and burnout in the teaching profession: European studies, issues, and research perspectives. Em R. Vanderbergue \& A. Huberman (Orgs.), Understanding and preventing teacher burnout: A source book of international practice and research (pp. 38-58). Cambridge: Cambridge University Press.

Russell, D. W., Altmaier, E., \& Van Velzen, D. (1987). Jobrelated stress, social suport, and burnout among classroom teachers. Journal of Applied Psychology, 72, 269-273.

Salanova, M., \& Llorens, S. (2008). Estado actual y retos futuros en el estudio del burnout. Papeles del Psicólogo, 29(1), 59-67.

Schwab, R. L., \& Iwanicki, E. F. (1982). Who are our burned out teachers? Educational Research Quaterly, 7(2), 5-16.

Seligmann-Silva, E. (1994). Desgaste mental do trabalhador dominado. Rio de Janeiro: Cortez.

Schaufeli, B. W., Leiter, M. P., \& Maslach, C. (2009). Burnout: 35 years of research and practice. Career Development International, 14(3), 204-220.

Silva, G. N., \& Carlotto, M. S. (2003). Síndrome de burnout: Um estudo com professores da rede pública. Revista de Psicologia Escolar e Educacional, 7(2), 145-153.

Sparks, D. (1983). Practical solutions for teacher stress. Theory Into Practice, 22(1), 3-44.

Woods, P. (1999). Intensification and stress in teaching. Em R. Vanderbergue \& M. A. Huberman (Orgs.), Understanding and preventing teacher burnout: A source book of international practice and research (pp. 115-138). Cambridge: Cambridge University Press.

Yong, Z., \& Yue, Y. (2007). Causes for burnout among secondary and elementary school teachers and preventive strategies. Chinese Education and Society, 40(5), 78-85.

Recebido: 18/01/2008 Última revisão13/05/2010 Aceite final: 20/05/2010 


\section{Sobre as autoras:}

Cristina Delgado Rodrigues: Psicóloga pela Universidade Luterana do Brasil - ULBRA/Canoas.

Laura Brandão Chaves: Psicóloga pela Universidade Luterana do Brasil - ULBRA/Canoas; Especialista clínica em Psicoterapia Cognitivo-Comportamental pela WP de Porto Alegre.

Mary Sandra Carlotto: Psicóloga; Mestre em Saúde Coletiva (ULBRA/RS); Doutora em Psicologia Social (USC/ES); Professora e Pesquisadora do Laboratório de Ensino e Pesquisa em Psicologia do Curso de Psicologia - Universidade Luterana do Brasil ULBRA/Canoas.

Endereço para correspondência: Cristina Delgado Rodrigues - Rua Monsenhor Veras, 412 - apto. 302 - Bairro Santana - 90610-010 Porto Alegre/RS. Endereço eletrônico: crisdr@via-rs.net.

Laura Brandão Chaves - Rodovia SC 434, km 14, Araçatuba - 88780-000 Imbituba/SC.

Endereço eletrônico: lauraibiraquera@ @otmail.com 